\title{
Relación entre factores psicosociales y el clima laboral en los integrantes de una orquesta sinfónica del Perú
}

\author{
Relationship between psychosocial factors and work environment in \\ the members of a Peruvian symphony orchestra
}

\section{Anthony Cristian Medina Paredes'}

\section{Revista \\ Educación y Sociedad}

Citar como: Medina Paredes, A. C. (2021). Relación entre factores psicosociales y el clima laboral en los integrantes de una orquesta sinfónica del Perú. Revista Educación y

Sociedad, 2(4), 58-68.

https://doi.org/10.53940/reys.v2i4.75

Artículo recibido: 06-07-2021 Artículo aprobado: 02-12-2021 Arbitrado por pares

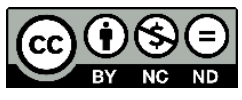

\section{ACEES}

\section{Resumen}

Esta investigación pretende hallar la influencia de los factores psicosociales considerados en el clima laboral; estableciéndose la hipótesis de que los factores psicosociales en estudio influyen significativamente en la percepción del clima laboral en una organización musical sin fines de lucro. Para este propósito se asumió una investigación observacional, cuantitativa, de corte transeccional y correlacional. Se estudió a una población de 41 músicos integrantes de una Orquesta Sinfónica del Sur del Perú. Los resultados de la investigación dan a conocer que existe una correlación positiva, moderada y significativa.

Palabras clave: factores psicosociales, clima laboral, salud laboral, seguridad ocupacional, condiciones laborales

\section{Abstract}

This research aims to find the influence of psychosocial factors taken into account in the work environment establishing the hypothesis that the psychosocial factors studied have a significant influence on the work environment perception in a non-profit music organization. For this purpose, an observational, quantitative, cross-sectional and correlational research was undertaken. A population of 41 musicians from a Symphonic Orchestra of Southern Peru was studied. The research results prove that there is indeed significant, positive and moderate correlation that means the more favorable the psychosocial factors.

Key words: psychosocial factors, work environment, occupational health, occupational security, working conditions 


\section{Introducción}

El desarrollo de los estudios en recursos humanos ha permitido enfocarnos en el ser humano como persona y su relación con un entorno organizacional. Gracias a esto se han desarrollado conceptos como el de calidad de vida en el trabajo tomada como base para alcanzar objetivos. Del mismo modo, hay que tener en cuenta que el individuo se encuentra inmerso social y psicológicamente en su trabajo y, al lograr optimizar el potencial humano, se impulsará en gran medida la competitividad de toda la organización (Chiavenato, 2007). De otro lado, existen diferentes factores laborales que pueden ocasionar enfermedades o lesiones físicas denominados riesgos laborales que, en muchas ocasiones, no son detectables a simple vista al generarse inadvertidamente en el día a día de la empresa como el estrés, la depresión y el agotamiento laboral (Dessler, 2015).

El eje central de esta investigación es la teoría general del estrés de la cual se desglosan dos modelos; el modelo demanda-control-apoyo social de Karasek (1979) y el modelo del desequilibrio esfuerzorecompensa de Siergrist (1996). Desde este punto de vista el estrés se presenta como una serie de respuestas de la persona ante exigencias del exterior que generan un desequilibrio entre lo que se demanda en relación a nuestra capacidad de respuesta. Asimismo, se ha considerado que el estrés está relacionado con la presencia de factores psicosociales de riesgo en las organizaciones (Osorio, 2017).

El objetivo general del presente estudio fue determinar la influencia de los factores psicosociales en la percepción del clima laboral en una organización musical sin fines de lucro de Arequipa, 2019, en esta oportunidad, las unidades de estudio fueron los músicos de la Orquesta Sinfónica del Sur del Perú (OSS).

\section{Aproximación conceptual a los factores psicosociales}

En 1984 mediante el documento oficial titulado "Factores Psicosociales en el Trabajo: Naturaleza, Incidencia y Prevención" la Organización Internacional del Trabajo [OIT] afirma que los factores psicosociales en el trabajo son interacciones entre el trabajo, su medio ambiente, la satisfacción en el trabajo y las condiciones de la organización. También, comprenden las capacidades del trabajador, sus necesidades, su cultura y su situación personal fuera del trabajo; lo cual puede influir en la salud, en el rendimiento y en la satisfacción en el trabajo.

Los factores psicosociales también se definen como riesgos de índole psicosocial que afectan la salud laboral, estos se originan en la manera de organizar el trabajo y precisamente en las condiciones de trabajo, teniendo consecuencias fisiológicas como los efectos neuroendocrinos; emocionales como la depresión, la ansiedad y el estrés; cognitivas como el bloqueo del aprendizaje, la restricción de la creatividad o la dificultad al momento de toma de decisiones; y conductuales como la adopción de actitudes violentas o la incursión en determinados vicios como el tabaco, drogas o alcohol (Moncada et al., 2014).

Cabe resaltar que para fines prácticos se pueden utilizar los términos "Factores Psicosociales", "Factores Psicosociales de Riesgo" y "Riesgos Psicosociales" indistintamente en este trabajo debido a que en la literatura han sido encontrados como términos intercambiables. Adicionalmente, advertimos que en la historia de este constructo se ha hecho más hincapié a su connotación negativa que en la positiva; por lo que al referirse a factores psicosociales se suele entender que son aquellos que pueden representar riesgos a la salud laboral (Moreno, 2011).

\section{Gestión internacional de la salud y seguridad ocupacional}

Actualmente se menciona como algo imprescindible que todas las empresas desarrollen programas de Salud y Seguridad Ocupacional (OHS) en relación con las normativas y parámetros internacionales que funcionan como marcos de referencia para mejorar la gestión de la salud y seguridad ocupacional de cada empresa; tal es el caso de las normas OHSAS (Occupational Health and Safety Assesment Series) 
(Sociedad Nacional de Minería, Petróleo y Energía [SNMPE], 2009) y el "Plan de Acción sobre la Salud de los Trabajadores" que corresponde al periodo 2015-2025 presentada por la Organización Mundial de la Salud y Organización Panamericana de la Salud (OMS y OPS, 2015).

\section{Globalización y riesgos laborales}

La globalización suele aportar mayores oportunidades para el desarrollo personal y profesional de un trabajador, sin embargo, también ocasiona mayores niveles de estrés, sobrecargas, conflictos y ambigüedades en las tareas o roles. Así, el outsourcing se va incrementado cada vez más por las crecientes exigencias del mundo actual como la calidad de los servicios, niveles cambiantes de demanda y la satisfacción del cliente, esto da como resultado un mayor grado de inseguridad laboral (Moreno y Garrosa, 2009).

\section{Factores y dimensiones psicosociales}

Importantes investigaciones han logrado establecer el número de factores psicosociales en base a las teorías "Demanda-Control” de Karasek (1979) y "Esfuerzo-Recompensa" de Siegrist (1996). Este es el caso de una revisión sistemática de la literatura publicada en la revista de Medicina Ocupacional de la Universidad de Oxford en la cual se identificaron 7 de 2426 investigaciones acerca de factores psicosociales y estrés en las cuales se descubrió que las altas demandas de trabajo, el bajo control en el trabajo, el bajo apoyo social del compañero, el bajo apoyo social del supervisor, los bajos niveles de justicia y un fuerte desbalance esfuerzo-recompensa están altamente relacionados con incrementos en los niveles de estrés (Nieuwenhuijsen et al., 2010).

De esta manera, y en virtud a lo mencionado, se han logrado estandarizar e incluir las dimensiones mencionadas en el anterior párrafo en seis factores psicosociales contenidos en la versión en español del cuestionario COPSOQ; el cual representa una de las herramientas más importantes y estandarizadas para el estudio de factores psicosociales considerados a cualquier tipo de empresa (Useche et al., 2019).

Con este sustento, se procedió a utilizar los factores psicosociales de la herramienta COPSOQ ISTAS 21 como sub variables pertenecientes al constructo psicosocial en estudio, estas son: exigencias psicológicas del trabajo; conflicto trabajo-familia; control sobre el trabajo; apoyo social y calidad de liderazgo; compensaciones del trabajo; y el capital social (Moncada et al., 2014). Al mismo tiempo, cada sub variable o factor psicosocial agrupa un determinado número de dimensiones como se expresa a continuación.

\section{Aproximación conceptual al clima laboral}

Existen muchos conceptos a cerca del clima laboral, sin embargo, en la mayoría de investigaciones modernas, se aborda esta variable como las impresiones de los miembros de una organización hacia sus dimensiones específicas. En líneas generales, el clima laboral se puede definir como el conjunto de percepciones compartidas acerca de las prácticas, políticas y procedimientos de la empresa que pueden ser informales o formales (Ployhart et al., 2014).

Por lo tanto, un clima organizacional óptimo se da en una empresa con un alto grado de colaboración y esfuerzos para facilitar el logro de los objetivos. Contrario a esto, y en el lado opuesto, el aislamiento y el alto grado de riesgos e inseguridades serían los causantes de un clima laboral inadecuado (Palma, 2004). El estado del clima laboral en una institución se manifiesta mediante las percepciones individuales subjetivas que en su conjunto van a definir operacionalmente el clima laboral de todo un grupo (Hons, 2004).

Arboccó (2009) ahonda en una serie de variables que son las que configuran el clima organizacional y sobre las que se van a hacer las percepciones. Estas son: el ambiente físico, las variables estructurales, el ambiente social, las variables personales y las variables propias del comportamiento organizacional. A 
partir de estas variables se desprenden las diferentes dimensiones que dan lugar a la evaluación del clima laboral de una institución.

\section{Dimensiones del clima laboral}

Palma (2004) establece cinco factores que determinan el clima laboral como la autorrealización personal y profesional, el involucramiento laboral, la supervisión, la comunicación y las condiciones laborales. Es importante señalar, que existe una ligera diferencia a nivel conceptual entre clima laboral y clima organizacional. El clima laboral hace referencia a la calidad de vida en el interior de una empresa y está determinada por diversos factores, es decir, un conjunto de características tanto tangibles como intangibles de una empresa con la capacidad de influir en la conducta de los trabajadores (Arboccó, 2009).

Por otro lado, se habla de clima organizacional cuando les otorgamos a estas características una medida perceptiva a nivel de la organización y de sus unidades en base a cómo actúan estos factores con los empleados y la sociedad. También, se consideran actores importantes en esta percepción la estructura organizacional y sus procesos; los mismos que van a influir en la psicología del individuo dando como resultado su precepción (Brunet, 2007).

Entonces, se podría decir que ambos términos a pesar de tener una pequeña diferencia, están relacionados y para hacer un análisis es necesario hablar de los dos. Esto debido a que el clima laboral es un término tanto objetivo como subjetivo al estar relacionado con la calidad de vida laboral; constructo que se configura gracias a las condiciones laborales como la seguridad e higiene (parte objetiva) y a la forma que el trabajador experimenta estas condiciones (parte subjetiva). Por otro lado, el clima organizacional que también es dependiente de las variables subjetivas mostradas líneas arriba se enfocan principalmente en la parte subjetiva ya que engloba percepciones y experiencias (Bordas, 2016).

\section{Metodología}

El diseño general de la investigación corresponde a un enfoque cuantitativo, de naturaleza observacional y de corte transaccional porque la recolección de datos se realizó en un único espacio de tiempo. Se apeló a la correlación como técnica estadística atendiendo a las variables factores psicosociales y clima laboral.

Las unidades de análisis de esta investigación son los músicos de la OSS y debido a la magnitud de la población la recolección de datos se realizó en la totalidad de sus integrantes activos (41 músicos). Los cuestionarios fueron aplicados bajo consentimiento informado y adicionalmente a esto se realizó una entrevista al director de dicha agrupación.

De acuerdo con el método de recolección de datos se utilizaron pruebas estandarizadas para la medición específica de los factores psicosociales y el clima laboral mediante cuestionarios (Hernández et al., 2014).

El instrumento utilizado para medir la variable Factores Psicosociales fue el Copenhagen Psychosocial Questionnaire (COPSOQ). Este es un cuestionario desarrollado en 1997 por el Departamento Psicosocial del Instituto Nacional de Salud Laboral de Dinamarca (AMI) posee tres versiones: la versión larga adecuada para la investigación científica; la media para ser usada por profesionales de clima organizacional; y, la versión corta para centros de trabajo (Kristensen et al., 2005). Cabe precisar que el COPSOQ se adaptó por primera vez al español el 2003 por cuenta del Instituto Sindical de Trabajo, Ambiente y Salud de España (ISTAS) tomando el nombre de CoPsoQ-istas21 y presentado como una herramienta para trabajar en el diagnóstico y prevención de riesgos psicosociales laborales.

EI ISTAS elaboró en el 2014 la segunda actualización del COPSOQ en español el cual se usó en esta investigación dado que es la más reciente y cuenta con óptimas propiedades psicométricas. En este sentido. Para efectos de esta investigación se usó la versión media ya que, según las especificaciones del 
instrumento, es la recomendada para evaluar centros de trabajo de más de 25 trabajadores (Moncada et al., 2013).

Para medir el clima laboral esta variable se usó la Escala de Clima Laboral de Sonia Palma Carrillo (CLSPC). Este cuestionario fue desarrollado en el año 2004 y aplicado en Lima Metropolitana a 1323 trabajadores dependientes laboralmente. De manera especial, este instrumento está enfocado en encontrar factores que integran percepciones de los colaboradores hacia el trabajo y, al mismo tiempo, permite diagnosticar la percepción del clima laboral individual y grupal.

EI CL-SPC posee cinco factores tales como autorrealización, involucramiento laboral, supervisión, comunicación y condiciones laborales. La puntuación de estos ítems posee un total de 250 en general y a cada factor le corresponde un puntaje de 50 como máximo. Ambos instrumentos apelan a ítems tipo Likert. Véase la Tabla B del anexo las categorías diagnósticas de este instrumento.

\section{Resultados}

\section{Distribuciones porcentuales de frecuencia}

En cuanto a la clasificación poblacional por sexo se observa una predominancia del sexo masculino en la OSS; en donde el 78,4\% ( $n=29)$ fueron hombres y el 21,6\% $(n=8)$ mujeres. Por otra parte, se observó que la mayoría de músicos tiene entre 31 y 45 años, es así que la distribución de frecuencias por edad indica que el 10,8\% ( $n=4)$ de los encuestados tiene menos de 31 años, el $56,8 \%(n=21)$ tiene entre 31 y 45 años, el $32,4 \%(n=12)$ tiene más de 45 años.

De acuerdo con la distribución de frecuencias por departamento/sección se indica que el $67,6 \%(n=25)$ de los encuestados ejecuta instrumentos de cuerda frotada, el $18,9 \%(n=7)$ de los encuestados ejecuta instrumentos de viento madera, el 10,8\% ( $n=4)$ ejecuta instrumentos de viento metal y el $2,7 \%(n=1)$ ejecuta un instrumento de percusión. Finalmente, de acuerdo con la distribución porcentual por puesto de trabajo, se observó que el $69,9 \%(n=24)$ de los encuestados se desempeña solo como músico, el $21,6 \%$ $(n=8)$ son músicos y capos de fila a la vez, el $5,4 \%(n=2)$ son músicos y delegados a la vez y el $8,1 \%$ (3) son músicos, capos de fila y delegados a la vez. Cabe resaltar que no hay diferencias remunerativas en cada puesto de trabajo. En total respondieron el instrumento 37 músicos.

\section{Resultados descriptivos de factores psicosociales}

Todos los resultados que se explican líneas abajo están reflejados en la Tabla A de los anexos del presente artículo.

\section{Factor psicosocial exigencias psicológicas del trabajo}

Encontramos que todas las prevalencias a la exposición de cada dimensión tales como exigencias cuantitativas, ritmo de trabajo, exigencias emocionales y las exigencias de esconder emociones. Se aprecia que el $55,79 \%$ de los músicos se encuentra expuestos a una situación desfavorable o riesgosa para la salud, un $28 \%$ a una situación intermedia y un $15.54 \%$ a una situación favorable. Esta cifra indica que existe un alto grado de presión psicológica laboral en el músico.

Este resultado se presenta de esta forma ya que en la investigación se observó los músicos poseen el tiempo prudente para realizar las tareas asignadas sin excesivas exigencias cuantitativas, el ritmo de trabajo, aparte de presentarse en altos grados en muchos momentos, se extiende durante las jornadas laborales incrementando la intensidad del trabajo.

De acuerdo con las exigencias emocionales la mayoría de encuestados en varias ocasiones son partícipes de situaciones desgastadoras emocionalmente, sin embargo, casi nunca tienen que atender los 
problemas personales de los demás. Por otro lado, existe cierta necesidad para ocultar emociones en el grupo de trabajo debido a las exigencias de amabilidad e igualdad de trato, sin embargo, no representa una situación preocupante para el equipo.

\section{Factor psicosocial conflicto trabajo-familia (dimensión doble presencia)}

El factor psicosocial conflicto trabajo-familia y engloba a la doble presencia como única dimensión, estos conceptos básicamente hacen referencia a las necesidades de equilibrar el tiempo entre las responsabilidades familiares y laborales, en este caso, sí existen conflictos trabajo-familia en la OSS pero de forma moderada. Es así que se pudo observar que el $35.14 \%$ de los músicos se encuentran en una situación desfavorable, el $37.84 \%$ se encuentra en una situación media y el $27.03 \%$ en una situación favorable para la salud.

\section{Factor psicosocial control sobre el trabajo}

Representa el promedio de todas las prevalencias incluidas como influencia, posibilidades de desarrollo y sentido del trabajo; se puede apreciar que el $62.30 \%$ de los músicos se encuentra en una situación favorable para la salud, es decir poseen cierta autonomía y control sobre sus responsabilidades, el $20.69 \%$ se encuentra en una situación intermedia y el 17.01\% en una situación desfavorable.

Existe cierta influencia del músico al momento de decidir cómo realizar sus deberes, sin embargo, no posee mucha injerencia en las decisiones que afectan el trabajo general de todo el equipo. Se puede resaltar una alta percepción de posibilidades de desarrollo ya que la gran mayoría de músicos ven que su trabajo requiere iniciativa, permite que aprendan nuevas cosas, les da la posibilidad de mejorar y ofrece las condiciones para aplicar tanto sus conocimientos como habilidades. Asimismo, la gran mayoría de encuestados sienten que sus tareas tienen sentido, les parecen importantes y están comprometidos con su profesión; lo mencionado ayuda a incrementar sentido que otorgarían los músicos a su trabajo en general.

\section{Factor psicosocial apoyo social y calidad de liderazgo}

Resulta de los promedios de los resultados de sus dimensiones tales como claridad de rol, conflicto de rol, apoyo social de compañeros, sentimiento de grupo, apoyo social de superiores, calidad de liderazgo y previsibilidad. De esta manera, se puede observar la calidad de las relaciones tanto personales como emocionales que existe entre los músicos y sus superiores. Específicamente en este resultado general se puede observar que el $36.86 \%$ de los músicos se encuentra en una situación psicosocial desfavorable, el $44.30 \%$ está en una situación intermedia y el $18.84 \%$ en una situación favorable.

Disgregando las dimensiones correspondientes a este factor psicosocial se observó que los roles en la OSS son relativamente claros. Esto nos remite a la existencia de armonía entre las expectativas de organización y las del trabajador en cuanto a las actividades que este desempeña. Además, existe cierta disconformidad entre las tareas de la organización y las creencias o valores de los músicos ya que la mayoría de ellos realizan actividades que son aceptadas por algunos y por otros no; lo cual nos remitiría a conflictos de rol. No existe un óptimo apoyo social o ayuda mutua entre músicos. No obstante, si existe cohesión grupal dado que la mayoría de músicos perciben que forman parte de un colectivo y se sienten identificados con este.

Respecto al apoyo social de superiores la mayoría de músicos perciben que el director está dispuesto a escuchar sus problemas laborales. También, gran parte de los músicos afirmaron que en muchas ocasiones o siempre reciben ayuda, apoyo y hablan con el director sobre cómo realizar el trabajo. Complementando el resultado anterior en cuanto a la calidad de liderazgo la mayoría de los músicos percibieron que el jefe inmediato, es decir el director artístico, se asegura de las buenas oportunidades de desarrollo profesional para cada uno de ellos. 
La previsibilidad, que se refiere al flujo eficiente de la información para tener la flexibilidad y adaptabilidad, nos arroja resultados que indican que la mayoría de los músicos examinados percibe que la organización les informa con suficiente anticipación sobre decisiones importantes. Al mismo tiempo, consideraron que reciben toda la información necesaria para desenvolverse en el trabajo lo cual mejora el nivel de previsibilidad de los músicos de la OSS.

\section{Factor psicosocial compensaciones del trabajo}

Muestra el promedio de todas prevalencias de la exposición de cada dimensión incluida en este factor tales como el reconocimiento, la inseguridad sobre el empleo y la inseguridad sobre las condiciones de trabajo. Se aprecia, además, que el $16.67 \%$ de los músicos encuestados se encuentra en una situación desfavorable para la salud, el $28.70 \%$ se encuentra expuesto a una situación medianamente favorable y el $54.63 \%$ a una situación favorable, lo cual explica la medida en que el músico es recompensado adecuadamente por su esfuerzo.

Este resultado obedecería a que la mayoría de los músicos evaluados perciben un alto grado de reconocimiento de su trabajo que su esfuerzo es valorado y respetado por la dirección.

Por último, se encontró que la mayoría de músicos no se siente inseguro en su empleo; ya que casi no existe preocupación por si los despiden o les renuevan el contrato ni por la dificultad de encontrar trabajo ante el riesgo de desempleo. Asimismo, existe cierta estabilidad ante cambios en las condiciones fundamentales del trabajo como horarios, tareas, salario y lugar de trabajo.

\section{Factor psicosocial capital social}

Resulta de los promedios obtenidos de todas las prevalencias que pertenecen a este factor, tales como la confianza vertical, y la justicia. En este resultado general, se puede apreciar que el $12.12 \%$ de los músicos examinados se encuentra en una situación desfavorable para la salud, el $18.18 \%$ de los músicos se encuentra en una situación intermedia y el $69.70 \%$ se encuentra en una situación favorable para la salud. Es decir, perciben que existen condiciones sociales como la confianza y justicia para alcanzar los objetivos organizacionales.

Existe un alto grado de confianza vertical ya que los músicos perciben certeza de que las personas que ocupan cargos superiores van a actuar correctamente y sin aprovecharse de ellos. Por lo tanto, la mayoría de músicos en gran medida perciben que la Dirección Desconcentrada de Cultura confía en su buen trabajo y ellos también pueden confiar en la información procedente de las oficinas administrativas de esta dependencia. Adicionalmente la mayoría percibe a la Orquesta como un lugar de trabajo justo debido al alto grado de imparcialidad en cuanto al trato de los músicos.

\section{Resultados descriptivos de clima laboral}

El 9.09\% de los músicos encuestados consideró que el clima laboral en la OSS es desfavorable, el 51.52\% considera que no es favorable ni desfavorable, el 36.36\%, como favorable y el 3.03\% de los sujetos estimó que el clima laboral es muy favorable. Es así que más de la mitad de los músicos perciben que las prácticas y procedimientos de la empresa no son inadecuados. 


\section{Figura 1}

Clima Laboral

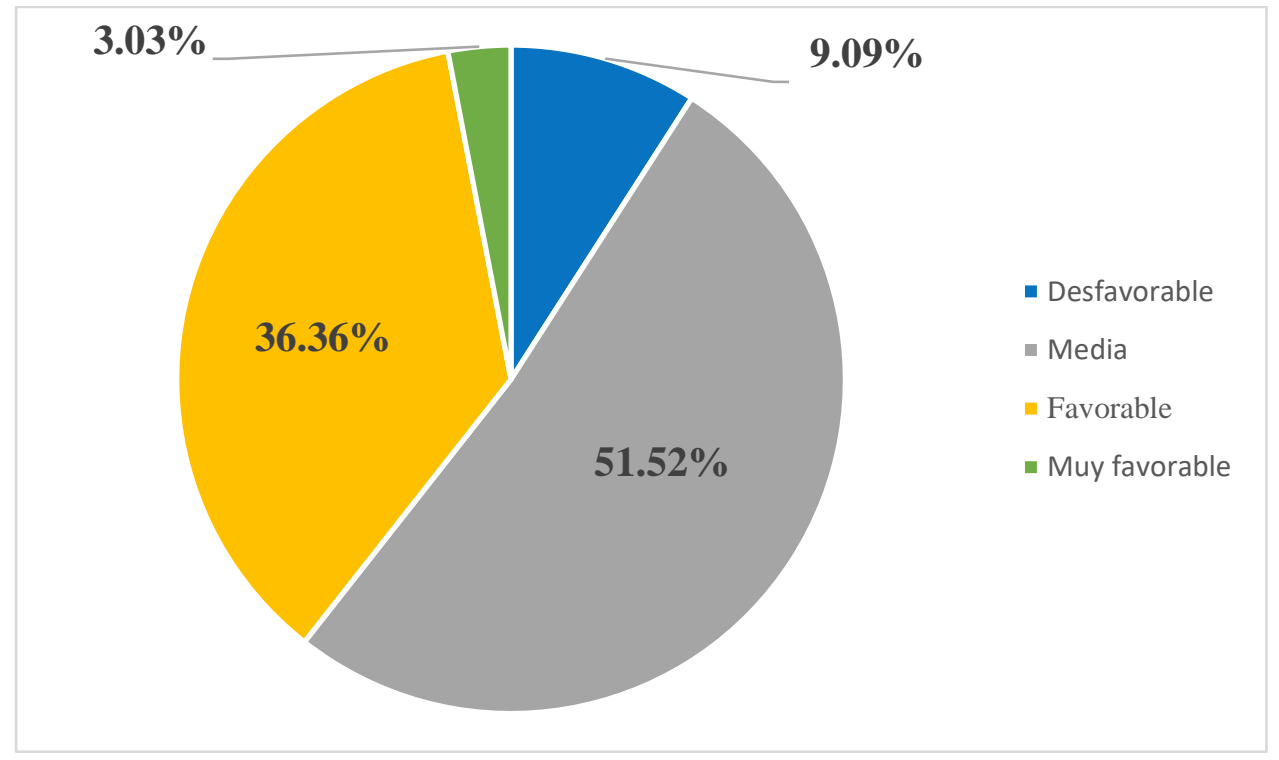

\section{Dimensión autorrealización}

El 2.94\% de los músicos considera que el nivel de autorrealización es muy desfavorable, el $14.71 \%$ considera que es desfavorable, el $47.06 \%$ considera que no es favorable ni desfavorable, el $32.35 \%$ lo considera favorable y el $2.94 \%$ de los músicos considera que el nivel de autorrealización es muy favorable. La mayoría de músicos percibe que el director se interesa mucho por el éxito de sus empleados. De acuerdo con las categorías diagnósticas de la herramienta el puntaje total obtenido para esta variable fue de 31 sobre 50.

\section{Dimensión involucramiento laboral}

Se traduce en el compromiso con el trabajo y los objetivos organizacionales, se aprecia que el $2.94 \%$ de los músicos evaluados considera un involucramiento laboral muy desfavorable, el $35.29 \%$ considera que no es favorable ni desfavorable, el $50 \%$ lo considera como favorable y el $11.76 \%$ percibe que el grado de involucramiento laboral es muy favorable. La mayoría de músicos se sienten muy comprometidos con el éxito de la organización, perciben que la realización de las labores cotidianas permite el desarrollo personal, y sienten que el cumplir con las actividades, su trabajo es una tarea estimulante. De acuerdo con las categorías diagnósticas de la herramienta, el puntaje total obtenido para esta variable fue de 35 sobre 50.

\section{Dimensión supervisión}

El grado de supervisión se expresa como el nivel de evaluación y apoyo a los músicos por parte de los superiores. Se aprecia así que el $2.94 \%$ de los músicos evaluados percibe un nivel de supervisión muy desfavorable, el $2.94 \%$ percibe esta dimensión como desfavorable, el $41.18 \%$ percibe que el grado de supervisión no es favorable ni desfavorable, el 50\% como favorable y por último se observa que un $2.94 \%$ de los músicos evaluados, perciben esta dimensión como muy favorable. La mayoría de los músicos evaluados de manera regular percibe que reciben la preparación necesaria para realizar el trabajo. De 
acuerdo con las categorías diagnósticas de la herramienta el puntaje total obtenido para esta variable fue de 33 sobre 50.

\section{Dimensión comunicación}

El 3.03\% de los músicos encuestados perciben que la comunicación en la organización es muy desfavorable, para el $21.21 \%$ es desfavorable, el $45.45 \%$ considera que la comunicación no es favorable ni desfavorable, el $27.27 \%$ considera que es favorable y el 3.03\% percibe la comunicación de la organización como muy favorable, es decir, sienten que existe eficiencia, coherencia y claridad en la transmisión de la información. De acuerdo con las categorías diagnósticas de la herramienta el puntaje total obtenido para esta variable fue de 30 sobre 50.

\section{Dimensión condiciones laborales}

Se refiere a la facilitación de recursos adecuados para la realización de las tareas diarias. Se observó que el $12.12 \%$ de los músicos examinados perciben condiciones laborables desfavorables, el $42.42 \%$, percibe condiciones laborales que no son favorables ni desfavorables, el 36.36\% siente que estas condiciones son favorables y por último el $9.09 \%$ considera que son muy favorables. La mayoría de los músicos examinados perciben en gran medida que sus colegas cooperan entre sí y que los objetivos de la empresa son retadores, sin embargo, perciben no disponer de la tecnología más adecuada. De acuerdo con las categorías diagnósticas de la herramienta el puntaje total obtenido para esta variable fue de 32 sobre 50.

\section{Resultados estadísticos y correlaciones}

La Tabla 1 nos muestra el grado de correlación existente entre Factores Psicosociales y Clima laboral; el cual es de 0.579 con una significancia de 0.00 que nos habla de una correlación positiva significativa entre las variables mencionadas.

\section{Tabla 1}

Valores de la correlación general entre variables

\begin{tabular}{lllll}
\hline & & \multicolumn{2}{l}{$\begin{array}{l}\text { Factores } \\
\text { Psicosociales }\end{array}$} & Clima Laboral \\
\hline Rho de Spearman & Factores Psicosociales & Coeficiente de correlación & 1,000 & 0,579 \\
& & Sig. (bilateral) & $\cdot$ &, 000 \\
& $\mathrm{~N}$ & 37 & 34 \\
\cline { 2 - 4 } & Clima Laboral & Coeficiente de correlación & 0,579 & 1,000 \\
& Sig. (bilateral) &, 000 &. \\
& $\mathrm{~N}$ & 34 & 34 \\
\hline
\end{tabular}

\section{Conclusiones}

Se concluye que los factores psicosociales se correlacionan de manera significativa y positiva con en el clima laboral de la organización musical sin fines de lucro estudiada. Del mismo modo, se puede observar un alto grado de exigencias psicológicas del trabajo en los músicos de la OSS al encontrarse que 
el $55.79 \%$ de ellos estiman una situación desfavorable para la salud debido al elevado ritmo de trabajo y exigencias emocionales. De igual modo, se evidenció un alto grado de control sobre el trabajo en dicha OSS. Asimismo, encontramos que el $62.30 \%$ de los músicos percibieron de manera favorable las posibilidades de desarrollo y sentido del trabajo al integrar esta orquesta.

También, se presenció que los sujetos consideraron como favorables las compensaciones del trabajo asociado a los altos niveles de reconocimiento y poca inseguridad sobre el empleo. En cuanto al capital social encontramos que los músicos percibieron situaciones favorables en gran medida y vinculadas a un alto grado de confianza vertical y justicia. Por otro lado, no existen conflictos trabajo-familia importantes dado que los músicos estudiados se encuentran casi uniformemente distribuidos entre un ambiente psicosocial favorable, intermedio y desfavorable.

Por último, y en referencia a los factores psicosociales, no se encontraron altos grados de apoyo social y calidad de liderazgo. También, evidenciamos altos grados de conflictos de rol y un bajo apoyo social de colegas.

Finalmente, y en cuanto al clima laboral se evidenció una situación intermedia a la luz de las respuestas de los integrantes de la OSS. En este mismo marco encontramos niveles intermedios en cuanto a la autorrealización, supervisión, comunicación y condiciones laborales. El grado de involucramiento denotó una consideración favorable.

\section{Referencias}

Arboccó, M. (2009). Recursos Humanos. Fondo Editorial de la Universidad Inca Garcilaso de la Vega.

Bordas, M. (2016). Gestión Estratégica del Clima Laboral. Universidad Nacional de Educación a Distancia.

Brunet, L. (2007). El Clima de Trabajo en las Organizaciones. Definición, diagnóstico y consecuencias .Trillas.

Chiavenato, I. (2007). Administración de Recursos Humanos. McGraw-Hill.

Dessler, G. (2015). Administración de Recursos Humanos (14 Ed.). Naucalpan de Juárez: Pearson.

Hernández, R., Fernández, C., y Baptista, M. (2014). Metodología de la Investigación. Mc Graw-Hill.

Hons, A. (2004). The Validation of an Organisational Climate Questionnaire in a Corporate Pharmacy Group [Not published master thesis]. North-West University .

Kristensen, T., Hannerz, H., Hogh, A., y Borg, V. (2005). The Copenhagen Psychosocial Questionnaire-a tool for the assessment. Scandinavian Journal of Work, Environment and Health, 438-449.

Moncada, S., Llorens, C., Andrés, R., y Moreno, N. y. (2014). Manual del método CoPsoQ-istas21 (versión 2) para la evaluación y la prevención de los riesgos psicosociales. Instituto Sindical de Trabajo, Ambiente y Salud.

Moncada, S., Utzet, M., Molinero, E., Llorens, C., Moreno, N., Galtés, A., y Navarro, A. (2013). The Copenhagen Psychosocial Questionnaire II (COPSOQ II) in Spain-A Tool for Psychosocial Risk Assessment at the Workplace. American Journal of Industrial Mdicine, 1-11.

Moreno, B. (2011). Factores y Riesgos Laborales Psicosociales: Conceptualización, Historia y Cambios Actuales. Medicina y Seguridad del Trabajo, 4-19.

Moreno, B., y Garrosa, E. (2009). Globalización y Riesgos Laborales Emergentes (Prefacio). Ciencia y Trabajo, A31-A34.

Nieuwenhuijsen, K., Bruinvels, D., y Frings, M. (2010). Psychosocial Work Environment and Stress-Related Disorders, a Systematic Review. Occupational Medicine, 277-286.

Organización Internacional del Trabajo. (1984). Factores Psicosociales en el Trabajo: Naturaleza, Incidencia y Prevención. Oficina Internacional del Trabajo Ginebra.

Organización Mundial de la Salud y Organización Panamericana de la Salud. (2015). Plan de Acción sobre la Salud de los Trabajadores. OMS y OPS.

Osorio, J. (2017). Estrés Laboral: Estudio de Revisión. Perspectivas en Psicología, 81-90.

Palma, S. (2004). Escala Clima Laboral CL-SPC. Manual. Cartolan. 
Ployhart, R., Hale, D., y Michael, C. (2014). Staffing Within the Social Context. En B. Schneider, \& K. Barbera (Ed.), The Oxford Handbook of Organizational Climate and Culture (pp. 23-43). Oxford University Press. Sociedad Nacional de Minería, Petróleo y Energía. (2009). Informe Quincenal de la SNMPE.

Useche, S., Montoro, L., Alonso, F., y Pastor, J. (2019). Psychosocial Work Factors, Job Stress and Strain at the Wheel: Validation of the Copenhagen Psychosocial Questionnaire (COPSOQ) in Professional Drivers. Frontiers in Psychology, 1-13. 